\title{
Active Interest Management for Improving Flow Completion Time in Named-Data Networking
}

\author{
Sugi Lee, Yusung Kim \\ Department of Computer \\ Engineering, Sungkyunkwan \\ University \\ Suwon, South Korea \\ sglee0323@gmail.com, \\ yskim525@gmail.com
}

\author{
Younghoon Kim \\ Department of Computer \\ Science, KAIST \\ Daejeon, South Korea \\ kyhoon@gmail.com
}

\author{
Ikjun Yeom \\ Department of Computer \\ Engineering, Sungkyunkwan \\ University \\ Suwon, South Korea \\ ijyeom@gmail.com
}

\begin{abstract}
Interest aggregation is the primary feature of named-data networking (NDN). The interest aggregation reduces duplicated transmissions for multiple requests on the same data, but can delay loss recovery when data packets are lost. Since retransmitted interest packets for loss recovery cannot be distinguished from regular interest packets, the lost data packets can be recovered after the timeout of the interest aggregation. The delayed loss recovery can largely increase flow completion time, particularly on small content downloads. In this paper, we propose an active interest management (AIM) scheme based on random early NACK to avoid the delayed loss recovery as well as keeping the benefit of interest aggregation. Using NS-3, we show that our scheme significantly reduced flow completion time of both small and large content.
\end{abstract}

\section{Categories and Subject Descriptors}

C.2.1 [Network Architecture and Design]: Network communications

\section{General Terms}

Performance

\section{Keywords}

Named-Data-Networking; Active Interest Management

\section{INTRODUCTION}

Named data networking (NDN) provides a content-centric communication model [7]. In NDN, content has its own name for routing and identifying. Consumers request content by sending request (interest) packets with content names, and the requested data packets are retrieved from original content providers or in-network caches. Using content names

Permission to make digital or hard copies of all or part of this work for persona or classroom use is granted without fee provided that copies are not made or distributed for profit or commercial advantage and that copies bear this notice and the full citation on the first page. Copyrights for components of this work owned by others than ACM must be honored. Abstracting with credit is permitted. To copy otherwise, or republish, to post on servers or to redistribute to lists, requires prior specific permission and/or a fee. Request permissions from permissions@acm.org.

CoNEXT Student Workshop'14, December 2, 2014, Sydney, Australia.

Copyright (C) 2014 ACM 978-1-4503-3282-8/14/12...\$15.00.

http://dx.doi.org/10.1145/2680821.2680830.

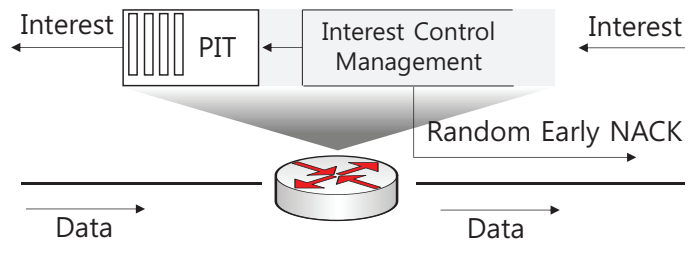

Figure 1: Proposed scheme

also facilitates interest aggregation. Interest aggregation enables forwarding of only one interest packet to an original server when multiple interest packets for the same data arrive in a short period of time. These interest packets are aggregated into an entry of pending interest table (PIT) in a NDN router. When the requested data packets arrive at the router, the data packets are forwarded to all interfaces from which the interest packets are received. The data packets are also stored in in-network caches in preparation for the next data request.

Using the features of NDN, we expect faster flow completion time. However, this paper indicates that delayed loss recovery is inherent in NDN due to the interest aggregation. Since retransmitted interest packets for loss recovery are not distinguished from regular (or previously aggregated) interest packets in PIT, the retransmitted interest packets will be also aggregated in the PIT. The consumer who experiences packet losses has to wait until the related PIT entries expire. The PIT entry timeout value should be reasonably large enough $^{1}$ to receive data packets which experience transient queuing delay, and the delayed loss recovery is unavoidable.

The delayed loss recovery can largely increase flow completion time, particularly on small content downloads. To improve flow completion time, we design an active interest management (AIM) scheme based on a random early negative-ACK (NACK), while keeping the benefit of the interest aggregation. Each NDN node controls the number of in-flight interest packets. Based on statistical probabilities, NACK packets are sent to consumers by consuming the interest information in PIT. In our evaluations using ndnSIM [2] as a NDN module of NS-3 [3], we show that the

\footnotetext{
${ }^{1}$ The default value of PIT entry timeout is two seconds in ndnSIM [2] and four seconds in CCNx [1]
} 
AIM scheme can significantly reduce flow completion time of both small and large content as much as using a RIO policy which requires additional DiffServ architecture.

\section{PROPOSED SCHEME}

AIM is similar to previous hop-by-hop interest management schemes [9][8], but we employ random early NACK as shown Figure 1. Every NDN node controls the size of PIT (the number of interest packets in flight) per outgoing interface. If node $\mathrm{A}$ forwards too many interest packets to node B, excess data packets from B to A will be dropped. Therefore, node A monitors the average PIT size, and sends NACK packets based on statistical probabilities to interfaces from which the interest packets are arrived. As the size of PIT grows, the probability for sending NACK grows too. Using the statistical probabilities is suitable to improve the flow completion time of small flows, since large flows have more chances to receive NACK packets due to their burstness. The NACK packets follow the reverse paths of the interest packets by consuming the interest information in PIT. Consumers increases the interest sending window by one if no timeout of interest ${ }^{2}$. When receiving NACK packets, the consumers reduce the interest sending rate by half, and retransmit the interest packets for the NACK packets. Since the NACK packet already consumed the previous interest packet in PIT, the first retransmitted interest packet can be forwarded (not be aggregated) to the original source.

\section{EVALUATION}

We compare our scheme with three different approaches;

- Basic: consumers use a simple interest control protocol such as ICP [4] and experience late loss recovery due to interest aggregation.

- No_Agg_RetInt: all configuration is same with 1) except NDN routers do not aggregate retransmitted interest packets (the retransmission information is marked by consumers).

- $\boldsymbol{R I O}$ : edge routers divide small and large content flows into two classes, and mark the class information on data packets' header. Core routers use different drop functions to give preferential treatment to small flow.

\subsection{Simulation Settings}

We conducted simulations using ndnSIM. In a simple dumbbell topology, RTT between consumers and original servers is $50 \mathrm{~ms}$. One in-network cache is located close to consumers. There is a bottleneck link (10 Mbps and 2ms RTT) between consumers and the cache. 10,000 small content items follows an exponential distribution with a mean $20 \mathrm{~KB}$, and 500 large content items follows pareto distribution with a mean $1 \mathrm{MB}$ (same configuration used in [5]). We generate requests 15 items per second for small flows and one item per second for large flow. The popularities follow Zipf distributions with $\alpha$ value of 0.8 for small content and 1.0 for large content. The timeout value of PIT entries was set to one second. The cache size was set to provide $40 \%$ hit ratio, and the queue size of bottleneck link was 1.5 times BDP. The configurations of $\boldsymbol{R I O}$ was the same as [6].

\footnotetext{
${ }^{2}$ The timeout of interest is usually much less than the timeout of PIT entry
}

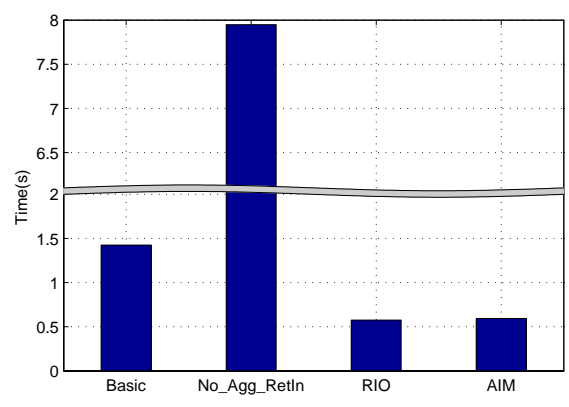

(a) On small content

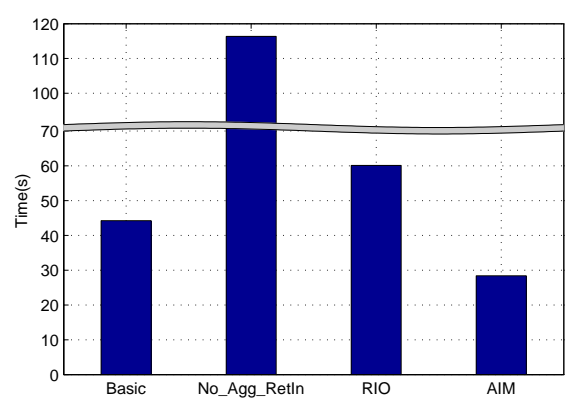

(b) On large content

Figure 2: Flow completion time

\subsection{Simulation results}

In Figure 2. shows the flow completion time of both small and large content. No_Agg_RetInt had the worse flow completion times for both small and large content. Transmitting data packets individually for all retransmission requests causes more severe congestion. To mitigate the situation, the timeout of interest packets should be adjusted properly such as random back-off. In $\boldsymbol{R I O}$, the flow completion time of small content was the lowest, but the flow completion time of large content was higher than that of Basic due to high priority on small flows. Our scheme showed the flow completion time of small content was reduced to as much as that of $\boldsymbol{R I O}$, while the flow completion time of large content was the lowest.

\section{CONCLUSION}

The interest aggregation feature of NDN provides a multicast nature to reduce duplicated content transmissions, but could delay the loss recovery time inherently. The delayed loss recovery can increase flow completion time, particularly on small flows. We design active interest management based on random early NACK in a hop-by-hop fashion. Without additional infrastructure, our scheme can solve the problem of the delayed loss recovery as keeping the benefit of interest aggregation.

\section{ACKNOWLEDGMENTS}

This work was supported by the National Research Foundation of Korea (NRF) (No. 2014R1A2A1A01004943) and the ICT R\&D program of MSIP/IITP. (No. 10041244) 


\section{REFERENCES}

[1] CCNx, http://www.ccnx.org/, 2014.

[2] ndnSIM, http://ndnsim. net/, 2014.

[3] NS-3, http://www. nsnam. org/, 2014.

[4] Giovanna Carofiglio, Massimo Gallo, and Luca Muscariello. ICP: Design and evaluation of an interest control protocol for content-centric networking, Computer Communications Workshops (INFOCOM WKSHPS), 2012 IEEE Conference on, pages 304-309. IEEE, 2012.

[5] Dinil Mon Divakaran. A spike-detecting AQM to deal with elephants, Computer Networks, 56(13):3087-3098, 2012.

[6] Liang Guo and Ibrahim Matta. The war between mice and elephants, Network Protocols, 2001. Ninth International Conference on, pages 180-188. IEEE, 2001.
[7] V. Jacobson, D.K. Smetters, J.D. Thornton, M.F Plass, N.H. Briggs, and R.L. Braynard. Networking named content, Proceedings of the 5th international conference on Emerging networking experiments and technologies, pages 1-12, ACM, 2009.

[8] Yaogong Wang, Natalya Rozhnova, Ashok Narayanan, David Oran, and Injong Rhee. An improved hop-by-hop interest shaper for congestion control in named data networking, Proceedings of the 3rd ACM SIGCOMM workshop on Information-centric networking, pages 55-60, ACM, 2013.

[9] Cheng Yi, Alexander Afanasyev, Ilya Moiseenko, Lan Wang, Beichuan Zhang, and Lixia Zhang. A case for stateful forwarding plane, Computer Communications, 36(7):779-791, 2013. 